\title{
Multibody Dynamics with Unilateral Constraints: Computational Modelling of Soft Contact and Dry Friction
}

\author{
Laetitia Paoli \\ Institut Camille Jordan, UMR CNRS 5208, \\ University of Lyon, University Jean Monnet, \\ 23 Rue Michelon, 42023 Saint-Etienne Cedex 2, France \\ laetitia.paoli@univ-st-etienne.fr
}

\begin{abstract}
We consider a system of rigid bodies subjected to unilateral constraints with soft contact and dry friction. When the constraints are saturated, velocity jumps may occur and the dynamics is described in generalized coordinates by a second-order measure differential inclusion for the unknown configurations. Observing that the right velocity obeys a minimization principle, a time-stepping algorithm is proposed. It allows to construct a sequence of approximate solutions satisfying at each time-step a discrete contact law which mimics the behaviour of the system in case of collision. In case of tangential contact, dry friction may lead to indeterminacies such as the famous Painlevé's paradoxes. By a precise study of the asymptotic properties of the scheme, it is shown that the limit of the approximate trajectories exhibits the same kind of indeterminacies.
\end{abstract}

Keywords: unilateral constraints, Coulomb's law, measure differential inclusion, time-stepping scheme

\section{Description of the Problem}

We consider a discrete mechanical system with $d$ degrees of freedom. We denote by $q \in \mathbb{R}^{d}$ its representative point in generalized coordinates and by $M(q)$ its inertia operator. We assume that the system is subjected to unilateral constraints characterized by the geometrical inequality

$$
g(q) \leq 0 \quad \text { (non penetration condition) }
$$

with a smooth (at least $C^{1}$ ) function $g$ such that $\nabla g$ does not vanish in a neighborhood of $\left\{q \in \mathbb{R}^{d} ; g(q)=0\right\}$. Let us denote by $\langle\cdot, \cdot\rangle$ the Euclidean inner product in $\mathbb{R}^{d}$. If for some instant $t>0$ the constraints are saturated, i.e. $g(q(t))=0$, then

$$
\left\langle\dot{q}^{-}(t), \nabla g(q(t))\right\rangle \geq 0, \quad\left\langle\dot{q}^{+}(t), \nabla g(q(t))\right\rangle \leq 0
$$


and the velocity may be discontinuous. It follows that $u=\dot{q}$ is a function of Bounded Variations and the dynamics is described by the Measure Differential Equation

$$
M(q) d u=f(t, q, u) d t+r
$$

where $d u$ is the Stieljes measure associated to $u$ and $r$ is the reaction due to the constraints. Of course a reaction is applied to the system only when a contact occurs and we have a complementarity condition

$$
g(q(t))<0 \Longrightarrow r=0
$$

Furthermore, we assume that the contact is non-adhesive which yields

$$
\langle r, \nabla g(q(t))\rangle \leq 0 \quad \text { if } g(q(t))=0
$$

In the frictionless case we get

$$
r \in-N(q(t)) \text { if } g(q(t))=0
$$

where $N(q(t))$ is the normal cone to the set of admissible configurations at $q(t)$ and in the frictional case

$$
r \in C(q(t)) \quad \text { if } g(q(t))=0
$$

where $C(q(t))$ is the so-called friction cone at $q(t)$. Hence we may rewrite (1) as a Measure Differential Inclusion ([17], [10])

$$
M(q) d u-f(t, q, u) d t \in \mathcal{R}(q)
$$

where

$$
\mathcal{R}(q)=\left\{\begin{array}{l}
\{0\} \quad \text { if } g(q)<0, \\
\mathbb{R}^{+}\left(n(q)+D_{1}(q)\right) \quad \text { if } g(q) \geq 0
\end{array}\right.
$$

and $n(q)=-\frac{\nabla g(q)}{\|\nabla g(q)\|}$ and $D_{1}(q)$ is the disc of center 0 and radius $\mu$ in $(\mathbb{R} n(q))^{\perp}$ with $\mu=0$ (frictionless constraints) or $\mu>0$ (Coulomb's friction).

Let us assume also soft contact i.e.

$$
u^{+}(t) \in T(q(t))=(\mathbb{R} n(q(t)))^{\perp} \quad \text { if } g(q(t))=0 .
$$

We infer that

$$
\left.u^{+}(t) \in\left(u^{-}(t)+M^{-1}(q(t)) \mathcal{R}(q(t))\right) \cap T(q(t))\right) \quad \text { if } g(q(t))=0 .
$$

In the frictionless case we may decompose $u^{ \pm}(t)$ as

$$
u^{ \pm}(t)=u_{N}^{ \pm}(t)+u_{T}^{ \pm}(t), \quad u_{N}^{ \pm}(t) \in \mathbb{R} M^{-1}(q(t)) n(q(t)), \quad u_{T}^{ \pm}(t) \in T(q(t)),
$$


with

$$
u_{N}^{ \pm}(t)=\frac{\left\langle u^{ \pm}(t), n(q(t))\right\rangle}{\left\langle n(q(t)), M^{-1}(q(t)) n(q(t))\right\rangle} .
$$

With (3)-(4) we get

$$
u_{N}^{+}(t)=0, \quad u_{T}^{+}(t)=u_{T}^{-}(t)
$$

which is equivalent to

$$
u^{+}(t)=\operatorname{Proj}_{q(t)}\left(0,\left(u^{-}(t)+M^{-1}(q(t)) \mathcal{R}(q(t))\right) \cap T(q(t))\right) \text { if } g(q(t))=0
$$

where $\operatorname{Proj}_{q(t)}$ denotes the projection relatively to the kinetic metric at $q(t)$.

In the frictional case, when $M(q) \equiv m \operatorname{Id}_{\mathbb{R}^{d}}, m>0$, Coulomb's law ([3], [11], [12]) yields

$$
-u^{+}(t) \in \partial \psi_{r_{N} D_{1}(q(t))}\left(r_{T}\right) \quad \text { if } g(q(t))=0
$$

where $r_{N}$ and $r_{T}$ are respectively the projection relatively to the Euclidean metric of $r$ on $\mathbb{R} n(q(t))$ and $T(q(t))$ and $\partial \psi_{r_{N} D_{1}(q(t))}$ is the indicatrix function of the disc of radius $\mu r_{N}$ and center 0 in $T(q(t))$. Reminding that $\mathcal{R}(q(t))=$ $\mathbb{R}^{+}\left(n(q(t))+D_{1}(q(t))\right),(5)$ is equivalent to

$$
-u^{+}(t) \in \operatorname{Proj}\left(T(q(t)), \partial \psi_{\mathcal{R}(q(t))}(r)\right)
$$

which can be rewritten as

$$
u^{+}(t)=\operatorname{Proj}\left(0,\left(u^{-}(t)+M^{-1}(q(t)) \mathcal{R}(q(t))\right) \cap T(q(t))\right) \text { if } g(q(t))=0 .
$$

Let us emphasize that (4) and (6) imply that $u^{+}(t) \neq u^{-}(t)$ only if $g(q(t))=$ 0 and $\left\langle u^{-}(t), n(q(t))\right\rangle<0$, i.e. only in case of collision. Moreover $u^{+}(t)$ is defined as the Argmin of the kinematically admissible right velocities. The same property holds when $M(q) \not \equiv m \operatorname{Id}_{\mathbb{R}^{d}}$ and $\mu>0$ and we still have ([12], [6])

$$
u^{+}(t)=\operatorname{Proj}_{q(t)}\left(0,\left(u^{-}(t)+M^{-1}(q(t)) \mathcal{R}(q(t))\right) \cap T(q(t))\right)
$$

if $g(q(t))=0$ and $\left.\left\langle u^{-}(t), n(q(t))\right)\right\rangle<0$. On the contrary, when $\left.g(q(t))\right)=0$ and $\left.\left\langle u^{-}(t), n(q(t))\right)\right\rangle=0,(4)$ yields

$$
u^{+}(t) \in u^{-}(t)+\left(M^{-1}(q(t)) \mathcal{R}(q(t)) \cap T(q(t))\right)
$$

and velocity jumps without collision may occur if $M^{-1}(q(t)) \mathcal{R}(q(t)) \cap T(q(t)) \neq$ $\{0\}$. Such phenomena can easily be observed when we consider the model problem of a slender rod in contact at one edge with an horizontal obstacle, leading to the famous Painlevé's paradoxes ([13], [14]): there exists a subset $\mathcal{A}\left(q(t), u^{-}(t)\right)$, containing $u^{-}(t)$ but not reduced to this single point, such that any value of $u^{+}(t) \in \mathcal{A}\left(q(t), u^{-}(t)\right)$ solves the problem (see for instance [8], [4], [1] or more recently [12], [2], [7]). Hence, in case of tangential contact with dry friction and non-trivial inertia operator, the dynamics exhibits indeterminacies. 


\section{Computational Modelling: the Contact Dynamics Approach}

In order to solve numerically the problem, the Contact Dynamics approach has been introduced by J.J. Moreau in the mid 80's ([10], [11], [12]). The core idea is to avoid any regularization of the unilateral constraints and to build a timestepping scheme by combining an Euler discretization of the measure differential inclusion (2) on each interval $\left[t_{i}, t_{i+1}\right]$ with an impulsional form of the contact law at $t_{i+1}$. More precisley the approximate position is updated as

$$
q_{i+1}=q_{i}+h u_{i}, \quad h=t_{i+1}-t_{i}
$$

and a "free" left velocity at $t_{i+1}$ is defined by

$$
v_{i+1}=u_{i}+h M^{-1}\left(q_{i+1}\right) f\left(t_{i+1}, q_{i+1}, u_{i}\right) .
$$

Then $u_{i+1}$ is the right velocity at $t_{i+1}$ given by

$$
M\left(q_{i+1}\right)\left(u_{i+1}-u_{i}\right)-h f\left(t_{i+1}, q_{i+1}, u_{i}\right) \in \mathcal{R}\left(q_{i+1}\right), \quad u_{i+1} \in T\left(q_{i+1}\right)
$$

and

$$
u_{i+1}=\mathcal{S}\left(q_{i+1}, v_{i+1}\right)
$$

where $\mathcal{S}$ is a discrete analogous of the contact law. Starting from the definition of $\mathcal{R}\left(q_{i+1}\right)$, we get immediately

$$
\mathcal{S}\left(q_{i+1}, v_{i+1}\right)=v_{i+1} \quad \text { if } g\left(q_{i+1}\right)<0 .
$$

If $g\left(q_{i+1}\right) \geq 0$ and $\left\langle v_{i+1}, n\left(q_{i+1}\right)\right\rangle>0$, the left velocity $v_{i+1}$ points inward and

$$
\mathcal{S}\left(q_{i+1}, v_{i+1}\right)=v_{i+1} .
$$

If $g\left(q_{i+1}\right) \geq 0$ and $\left\langle v_{i+1}, n\left(q_{i+1}\right)\right\rangle<0$, we may interpret $t_{i+1}$ as a collision instant and with (6) we get

$$
\mathcal{S}\left(q_{i+1}, v_{i+1}\right)=\operatorname{Proj}_{q_{i+1}}\left(0,\left(v_{i+1}+M^{-1}\left(q_{i+1}\right) \mathcal{R}\left(q_{i+1}\right)\right) \cap T\left(q_{i+1}\right)\right) .
$$

Finally, if $g\left(q_{i+1}\right) \geq 0$ and $\left\langle v_{i+1}, n\left(q_{i+1}\right)\right\rangle=0$, we get

$$
u_{i+1} \in v_{i+1}+\left(M^{-1}\left(q_{i+1}\right) \mathcal{R}\left(q_{i+1}\right) \cap T\left(q_{i+1}\right)\right) .
$$

Hence $u_{i+1}$ is uniquely defined if $M^{-1}\left(q_{i+1}\right) \mathcal{R}\left(q_{i+1}\right) \cap T\left(q_{i+1}\right)=\{0\}$ and we have $u_{i+1}=\mathcal{S}\left(q_{i+1}, v_{i+1}\right)=v_{i+1}$. On the contrary, if $M^{-1}\left(q_{i+1}\right) \mathcal{R}\left(q_{i+1}\right) \cap T\left(q_{i+1}\right) \neq$ $\{0\}, t_{i+1}$ may be interpreted as a discrete tangential contact with possible indeterminacies and there is not any natural choice for $u_{i+1}$.

In [10], [11], [12] J.J. Moreau proposed $u_{i+1}=\mathcal{S}\left(q_{i+1}, v_{i+1}\right)=v_{i+1}$ if $g\left(q_{i+1}\right) \leq 0,\left\langle v_{i+1}, n\left(q_{i+1}\right)\right\rangle=0$ and $M^{-1}\left(q_{i+1}\right) \mathcal{R}\left(q_{i+1}\right) \cap T\left(q_{i+1}\right) \neq\{0\}$. It follows that $\mathcal{S}$ is defined as

$$
\mathcal{S}\left(q, u^{-}\right)=\left\{\begin{array}{l}
v \quad \text { if } v \in V(q) \\
\operatorname{Proj}_{q}\left(0,\left(v+M^{-1}(q) \mathcal{R}(q)\right) \cap T(q)\right) \quad \text { otherwise }
\end{array}\right.
$$


with

$$
V(q)=\left\{\begin{array}{l}
\mathbb{R}^{d} \quad \text { if } g(q)<0, \\
\left\{v \in \mathbb{R}^{d} ;\langle v, n(q)\rangle \geq 0\right\} \quad \text { if } g(q) \geq 0 .
\end{array}\right.
$$

For this scheme the following stability property holds.

Proposition 1. For all $i \geq 0$, let $r_{i+1}=M\left(q_{i+1}\right)\left(u_{i+1}-u_{i}\right)-h f\left(t_{i+1}, q_{i+1}, u_{i}\right)$ and $\|\cdot\|_{q_{i+1}}$ be the kinetric norm at $t_{i+1}$ defined by $\|v\|_{q_{i+1}}=\left\langle v, M\left(q_{i+1}\right) v\right\rangle^{1 / 2}$ for all $v \in \mathbb{R}^{d}$. Then, $r_{i+1} \in \mathcal{R}\left(q_{i+1}\right),\left\langle u_{i+1}, r_{i+1}\right\rangle \leq 0$ and

$$
\left\|u_{i+1}\right\|_{q_{i+1}} \leq\left\|u_{i}\right\|_{q_{i+1}}+h\left\|M^{-1 / 2}\left(q_{i+1}\right)\right\|\left\|f\left(t_{i+1}, q_{i+1}, u_{i}\right)\right\| .
$$

Proof. If $r_{i+1}=0$ the result is obvious.

Otherwise, $v_{i+1} \notin V\left(q_{i+1}\right)$ and $\left\langle v_{i+1}, n\left(q_{i+1}\right)\right\rangle<0$. By definition of $\mathcal{S}$ we get

$$
u_{i+1}=\operatorname{Proj}_{q_{i+1}}\left(0,\left(v_{i+1}+M^{-1}\left(q_{i+1}\right) \mathcal{R}\left(q_{i+1}\right)\right) \cap T\left(q_{i+1}\right)\right) .
$$

Hence

$\left\langle-u_{i+1}, M\left(q_{i+1}\right)\left(v-u_{i+1}\right)\right\rangle \leq 0 \quad \forall v \in\left(v_{i+1}+M^{-1}\left(q_{i+1}\right) \mathcal{R}\left(q_{i+1}\right)\right) \cap T\left(q_{i+1}\right)$.

By choosing $v=v_{i+1}+\lambda M^{-1}\left(q_{i+1}\right) n\left(q_{i+1}\right)$ with

we obtain

$$
\lambda=-\frac{\left\langle v_{i+1}, n\left(q_{i+1}\right)\right\rangle}{\left\langle n\left(q_{i+1}\right), M^{-1}\left(q_{i+1}\right) n\left(q_{i+1}\right)\right\rangle}
$$

$$
\left\langle-u_{i+1}, M\left(q_{i+1}\right)\left(v_{i+1}-u_{i+1}\right)\right\rangle=\left\langle u_{i+1}, r_{i+1}\right\rangle \leq 0 .
$$

Furthermore

$$
\begin{aligned}
& \left\|u_{i+1}\right\|_{q_{i+1}} \leq\|v\|_{q_{i+1}} \leq\left\|v_{i+1}\right\|_{q_{i+1}}=\left\|u_{i}+h M^{-1}\left(q_{i+1}\right) f\left(t_{i+1}, q_{i+1}, u_{i}\right)\right\|_{q_{i+1}} \\
& \leq\left\|u_{i+1}\right\|_{q_{i+1}}+h\left\|M^{-1}\left(q_{i+1}\right) f\left(t_{i+1}, q_{i+1}, u_{i}\right)\right\|_{q_{i+1}} \\
& \leq\left\|u_{i+1}\right\|_{q_{i+1}}+h\left\|M^{-1 / 2}\left(q_{i+1}\right)\right\|\left\|f\left(t_{i+1}, q_{i+1}, u_{i}\right)\right\| .
\end{aligned}
$$

Observing that the inequality $\left\langle u_{i+1}, r_{i+1}\right\rangle \leq 0$ corresponds to a dissipativity property, we infer that the scheme reproduces at the discrete level the main features of the dynamics except the indeterminacies of Coulomb's law. Indeed, $\mathcal{S}(q, v)=v$ if $g(q)=0,\langle v, n(q)\rangle=0$ and the discrete contact law does not lead to any velocity jump in case of tangential contact.

Starting from Proposition 1, the convergence of the scheme has been established by M. Monteiro-Marques in [9] when $M(q) \equiv m \operatorname{Id}_{\mathbb{R}^{d}}(m>0)$ and $\mu \geq 0$ and by R. Dzonou and M. Monteiro-Marques in [5] when $M(q) \not \equiv m \operatorname{Id}_{\mathbb{R}^{d}}$ and $\mu=0$. In both cases we have $M^{-1}(q) \mathcal{R}(q) \cap T(q)=\{0\}$ for all $q \in \mathbb{R}^{d}$ such that $g(q)=0$ and the difficulty due to indeterminacies is avoided. Nevertheless the convergence has also been proved recently when $M(q) \not \equiv m \operatorname{Id}_{\mathbb{R}^{d}}$ and $\mu>0$ ([15]), so a natural question arises: is it possible to recover with such a scheme velocity jumps without collisions at the limit when $h$ tends to zero? A first answer has been given by J.J. Moreau in [12]: numerical simulations show that the approximated trajectories exhibit the plurality of solutions given by Coulomb's law. 


\section{Asymptotic Properties of the Discrete Contact Law}

Let us assume from now on that $M(q) \not \equiv m \operatorname{Id}_{\mathbb{R}^{d}}(m>0)$ and $\mu>0$. Then the limit trajectory will satisfy in case of convergence the following property

$$
u^{+}(t) \in \lim _{\varepsilon \rightarrow 0}\left\{\mathcal{S}\left(q_{\varepsilon}, u_{\varepsilon}^{-}\right) ; q_{\varepsilon} \in B(q, \varepsilon), u_{\varepsilon}^{-} \in B\left(u^{-}(t), \varepsilon\right)\right\}
$$

We may recover the indeterminacies of Coulomb's law if, for all $\left(q, u^{-}\right)$such that $g(q)=0,\left\langle u^{-}, n(q)\right\rangle=0$ and $M^{-1}(q) \mathcal{R}(q) \cap T(q) \neq\{0\}$, we have

$$
\lim _{\varepsilon \rightarrow 0} d_{H}\left(\mathcal{A}_{\varepsilon}\left(q, u^{-}\right), \mathcal{A}\left(q, u^{-}\right)\right)=0
$$

with

$$
\mathcal{A}_{\varepsilon}\left(q, u^{-}\right)=\left\{\mathcal{S}\left(q_{\varepsilon}, u_{\varepsilon}^{-}\right) ; q_{\varepsilon} \in B(q, \varepsilon), u_{\varepsilon}^{-} \in B\left(u^{-}, \varepsilon\right)\right\} .
$$

Let us recall that the Hausdorff distance between two subsets $A$ and $B$ of $\mathbb{R}^{d}$ is defined as

$$
d_{H}(A, B)=\max (e(A, B), e(B, A))
$$

where

$$
\begin{aligned}
& e(A, B)=\sup _{a \in A} \operatorname{dist}(a, B)=\sup _{a \in A} \inf _{b \in B}\|a-b\| \quad(\text { the excess of } A \text { from } B), \\
& e(B, A)=\sup _{b \in B} \operatorname{dist}(b, A)=\sup _{b \in B} \inf _{a \in A}\|b-a\| \quad(\text { the excess of } B \text { from } A) .
\end{aligned}
$$

Hence (8) can be decomposed as

$$
\lim _{\varepsilon \rightarrow 0} \sup \left\{\operatorname{dist}\left(\mathcal{S}\left(q_{\varepsilon}, u_{\varepsilon}^{-}\right), \mathcal{A}\left(q, u^{-}\right)\right) ; \quad q_{\varepsilon} \in B(q, \varepsilon), u_{\varepsilon}^{-} \in B\left(u^{-}, \varepsilon\right)\right\}=0
$$

which can be interpreted as an asymptotic consistency property of the discrete contact law $\mathcal{S}$ and

$$
\lim _{\varepsilon \rightarrow 0} \sup \left\{\operatorname{dist}\left(v, \mathcal{A}_{\varepsilon}\left(q, u^{-}\right)\right) ; v \in \mathcal{A}\left(q, u^{-}\right)\right\}=0
$$

which can be interpreted as an asymptotic indeterminacy of the scheme.

In the one-dimensional friction case, i.e. when $\operatorname{Dim}\left(\operatorname{Span}\left(D_{1}(q)\right)\right)=1$, then

$$
\mathcal{A}\left(q, u^{-}\right)=\left\{\begin{array}{l}
\left\{u^{-}, \tilde{u}\right\} \text { if } \min _{w \in D_{1}(q)}\left\langle n(q), M^{-1}(q)(n(q)+w)\right\rangle<0, \\
{\left[u^{-}, \tilde{u}\right] \text { if } \min _{w \in D_{1}(q)}\left\langle n(q), M^{-1}(q)(n(q)+w)\right\rangle=0}
\end{array}\right.
$$

with

$$
\tilde{u}=\operatorname{Proj}_{q}\left(0,\left(u^{-}+M^{-1}(q) \mathcal{R}(q)\right) \cap T(q)\right)
$$

(see [12]). Then we can prove that (9) is satisfied while (10) is not always true and depends on the evolution of the mappings $q_{\varepsilon} \mapsto \mathcal{R}\left(q_{\varepsilon}\right)$ and $q_{\varepsilon} \mapsto n\left(q_{\varepsilon}\right)$ in a neighborhood of the contact point. More precisely let us assume that 
(H1) the mapping $M$ is of class $C^{1}$ from $\mathbb{R}^{d}$ to the set of symmetric positive definite $d \times d$ matrices;

(H2) the function $g$ belongs to $C^{1}\left(\mathbb{R}^{d}\right), \nabla g$ is locally Lipschitz continuous and does not vanish in a neighbourhood of $\left\{q \in \mathbb{R}^{d} ; g(q)=0\right\}$;

(H3) for all $q \in \mathbb{R}^{d}, D_{1}(q)$ is a closed, bounded, convex subset of $\mathbb{R}^{d}$ such that $0 \in D_{1}(q)$ and the multivalued mapping $q \mapsto D_{1}(q)$ is Hausdorff continuous. Furthermore, $\nabla g(q) \notin \operatorname{Span}\left(D_{1}(q)\right)$ for all $q \in \mathbb{R}^{d}$ such that $\nabla g(q) \neq 0$.

We denote by $K$ the set of admissible configurations i.e.

$$
K=\left\{q \in \mathbb{R}^{d} ; g(q) \leq 0\right\} .
$$

Now let $\left(q, u^{-}\right) \in \mathbb{R}^{d} \times \mathbb{R}^{d}$ such that $g(q) \geq 0,\left\langle u^{-}, n(q)\right\rangle=0$ and $M^{-1}(q) \mathcal{R}(q) \cap$ $T(q) \neq\{0\}$. With assumption (H2) there exists $r_{q}>0$ such that the mapping

$$
n:\left\{\begin{array}{l}
\bar{B}\left(q, r_{q}\right) \rightarrow \mathbb{R}^{d} \\
q^{\prime} \mapsto n\left(q^{\prime}\right)=-\frac{\nabla g\left(q^{\prime}\right)}{\left\|\nabla g\left(q^{\prime}\right)\right\|}
\end{array}\right.
$$

is well defined and Lipschitz continuous. Let us assume moreover that, possibly reducing $r_{q}$, we have

(H4) $\operatorname{dim}\left(\operatorname{Span}\left(D_{1}\left(q^{\prime}\right)\right)\right)=1$ for all $q^{\prime} \in \bar{B}\left(q, r_{q}\right)$.

We may observe that $M^{-1}(q) \mathcal{R}(q) \cap T(q) \neq\{0\}$ if and only if there exists $w \in D_{1}(q)$ such that $\left\langle n(q), M^{-1}(q)(n(q)+w)\right\rangle=0$. Hence we introduce the mapping $\gamma: \bar{B}\left(q, r_{q}\right) \rightarrow \mathbb{R}$ defined by

$$
\gamma\left(q^{\prime}\right)=\min _{w^{\prime} \in D_{1}\left(q^{\prime}\right)}\left\langle n\left(q^{\prime}\right), M^{-1}\left(q^{\prime}\right)\left(n\left(q^{\prime}\right)+w^{\prime}\right)\right\rangle \quad \forall q^{\prime} \in \bar{B}\left(q, r_{q}\right) .
$$

With the previous assumptions we obtain that $\gamma$ is continuous at $q$ and $\gamma(q) \leq 0$. Then we have the following result:

Theorem 1. [16] If $\gamma(q)<0$ or $\gamma(q)=0$ and for all $\varepsilon \in\left(0, r_{q}\right)$ there exists $q_{\varepsilon} \in B(q, \varepsilon) \backslash(\operatorname{Int}(K) \cup\{q\})$ such that $\gamma\left(q_{\varepsilon}\right)>0$, we have

$$
\lim _{\varepsilon \rightarrow 0} d_{H}\left(\mathcal{A}\left(q, u^{-}\right), \mathcal{A}_{\varepsilon}\left(q, u^{-}\right)\right)=0 .
$$

Otherwise, if $\gamma(q)=0$ and there exists $\varepsilon_{q} \in\left(0, r_{q}\right)$ such that $\gamma\left(q^{\prime}\right) \leq 0$ for all $q^{\prime} \in B\left(q, \varepsilon_{q}\right) \backslash \operatorname{Int}(K)$, then $d_{H}\left(\mathcal{A}\left(q, u^{-}\right), \mathcal{A}_{\varepsilon}\left(q, u^{-}\right)\right)$does not tend to zero as $\varepsilon$ tends to zero if $\tilde{u} \neq u^{-}$and we only have

$$
\lim _{\varepsilon \rightarrow 0} d_{H}\left(\left\{u^{-}, \tilde{u}\right\}, \mathcal{A}_{\varepsilon}\left(q, u^{-}\right)\right)=0 .
$$

Idea of the proof: For all $\varepsilon \in\left(0, r_{q}\right)$ and $\left(q_{\varepsilon}, u_{\varepsilon}^{-}\right) \in B(q, \varepsilon) \times B\left(u^{-}, \varepsilon\right)$ such that $u_{\varepsilon}^{+}=\mathcal{S}\left(q_{\varepsilon}, u_{\varepsilon}^{-}\right) \neq u_{\varepsilon}^{-}$we have

$$
g\left(q_{\varepsilon}\right) \geq 0, \quad\left\langle u_{\varepsilon}^{-}, n\left(q_{\varepsilon}\right)\right\rangle<0
$$


and $u_{\varepsilon}^{+}=\tilde{u}_{\varepsilon}$ with

$$
\tilde{u}_{\varepsilon}=\operatorname{proj}_{q_{\varepsilon}}\left(0,\left(u_{\varepsilon}^{-}+M^{-1}\left(q_{\varepsilon}\right) \mathcal{R}\left(q_{\varepsilon}\right)\right) \cap T\left(q_{\varepsilon}\right)\right) .
$$

By using the same kind of arguments as in Proposition 1, we obtain that $\left(\tilde{u}_{\varepsilon}\right)_{r_{q}>\varepsilon>0}$ is bounded. Moreover we can decompose $\tilde{u}_{\varepsilon}$ as follows

$$
\tilde{u}_{\varepsilon}=u_{\varepsilon}^{-}+\lambda_{\varepsilon} M^{-1}\left(q_{\varepsilon}\right)\left(n\left(q_{\varepsilon}\right)+w_{\varepsilon}\right),
$$

with $\lambda_{\varepsilon}>0$ and $w_{\varepsilon} \in D_{1}\left(q_{\varepsilon}\right)$ such that

$$
\left\langle n\left(q_{\varepsilon}\right), M^{-1}\left(q_{\varepsilon}\right)\left(n\left(q_{\varepsilon}\right)+w_{\varepsilon}\right)\right\rangle=0
$$

for all $\varepsilon \in\left(0, r_{q}\right)$. Using assumption (H4) (or assumption (H'4) see below) we infer that there exists a unique vector $\tilde{w} \in D_{1}(q)$ such that $\left\langle n(q), M^{-1}(q)(n(q)+\right.$ $\tilde{w})\rangle=0$ and with assumptions (H1)-(H3) we obtain

$$
\lim _{\varepsilon \rightarrow 0} w_{\varepsilon}=\tilde{w} .
$$

Moreover, the boundedness of $\left(\tilde{u}_{\varepsilon}\right)_{r_{q}>\varepsilon>0}$ implies that $\left(\lambda_{\varepsilon}\right)_{r_{q}>\varepsilon>0}$ is also bounded and we infer that the adherence values of $\left(\tilde{u}_{\varepsilon}\right)_{r_{q}>\varepsilon>0}$ belong to $u^{-}+\left(M^{-1}(q) \mathcal{R}(q) \cap\right.$ $T(q))$.

If furthermore there exists $\varepsilon_{q} \in\left(0, r_{q}\right)$ such that $\gamma\left(q^{\prime}\right) \leq 0$ for all $q^{\prime} \in$ $B\left(q, \varepsilon_{q}\right) \backslash \operatorname{Int}(K)$, then we can prove that $\left(\tilde{u}_{\varepsilon}\right)_{r_{q}>\varepsilon>0}$ admits a unique adherence value given by $\tilde{u}$. It follows that

$$
\lim _{\varepsilon \rightarrow 0} d_{H}\left(\left\{u^{-}, \tilde{u}\right\}, \mathcal{A}_{\varepsilon}\left(q, u^{-}\right)\right)=0 .
$$

On the contrary, if for any $\varepsilon \in\left(0, r_{q}\right)$ there exists $q_{\varepsilon} \in B(q, \varepsilon) \backslash(\operatorname{Int}(K) \cup\{q\})$ such that $\gamma\left(q_{\varepsilon}\right)>0$, then, for any $\bar{v} \in\left[u^{-}, \tilde{u}\right] \backslash\left\{u^{-}\right\}$, $\operatorname{dist}\left(\bar{v}, \mathcal{A}_{\varepsilon}\left(q, u^{-}\right)\right)$tends to zero as $\varepsilon$ tends to zero. Indeed, we may construct a sequence $\left(q_{\varepsilon_{n}}, u_{\varepsilon_{n}}^{-}\right)_{n \geq 1}$ with $\left(\varepsilon_{n}\right)_{n>1}$ decreasing to zero such that $\left(q_{\varepsilon_{n}}, u_{\varepsilon_{n}}^{-}\right) \in B\left(q, \varepsilon_{n}\right) \times B\left(u^{-}, \varepsilon_{n}\right)$, and $\gamma\left(q_{\varepsilon_{n}}\right)>0$ for all $n \geq 1$ and $\left(\mathcal{S}\left(q_{\varepsilon_{n}}, u_{\varepsilon_{n}}^{-}\right)\right)_{n \geq 1}$ converges to $\bar{v}$. It follows that

$$
\lim _{\varepsilon \rightarrow 0} d_{H}\left(\left[u^{-}, \tilde{u}\right], \mathcal{A}_{\varepsilon}\left(q, u^{-}\right)\right)=0 .
$$

Then we conclude by using the continuity of $\gamma$ at $q$ and the definition of $\mathcal{A}\left(q, u^{-}\right)$.

We infer that the discrete contact law always satisfies the asymptotic consistency property while the asymptotic indeterminacy of the scheme holds only if $\gamma(q)<0$ or $\gamma(q)=0$ and for all $\varepsilon \in\left(0, r_{q}\right)$ there exists $q_{\varepsilon} \in B(q, \varepsilon) \backslash(\operatorname{Int}(K) \cup\{q\})$ such that $\gamma\left(q_{\varepsilon}\right)>0$ if $\tilde{u} \neq u^{-}$. In the latter case, any $\bar{v} \in\left[u^{-}, \tilde{u}\right] \backslash\left\{u^{-}, \tilde{u}\right\}$ is the limit of a sequence of post-collision velocities $\left(\mathcal{S}\left(q_{\varepsilon_{n}}, u_{\varepsilon_{n}}^{-}\right)=\tilde{u}_{\varepsilon_{n}}\right)_{n \geq 1}$ with $\left(\varepsilon_{n}\right)_{n \geq 1}$ decreasing to zero and $\left(q_{\varepsilon_{n}}, u_{\varepsilon_{n}}^{-}\right) \in B\left(q, \varepsilon_{n}\right) \times B\left(u^{-}, \varepsilon_{n}\right)$ for all $n \geq 1$. Hence, for all $n \geq 1, \mathcal{S}\left(q_{\varepsilon_{n}}, u_{\varepsilon_{n}}^{-}\right)=\tilde{u}_{\varepsilon_{n}}$ is defined as the Argmin of the kinetic 
norm of the admissible right velocities at $\left(q_{\varepsilon_{n}}, u_{\varepsilon_{n}}^{-}\right)$but $\bar{v}$ is not the Argmin of the kinetic norm of the admissible right velocities at $\left(q, u^{-}\right)$. It means that the minimization property (7) defining post-collision velocities is not continuous at $\left(q, u^{-}\right)$and it appears that this mathematical propery is deeply related to the indeterminacies of Coulomb's law.

Finally let us emphasize that (H3) allows to take into account both isotropic and anisotropic friction. Moreover the conclusions of Theorem 1 are still valid when (H4) is replaced by

(H'4) $D_{1}\left(q^{\prime}\right)$ is strictly convex for any $q^{\prime} \in \bar{B}\left(q, r_{q}\right)$ i.e. for any $w_{1}$ and $w_{2}$ belonging to $D_{1}\left(q^{\prime}\right)$ such that $w_{1} \neq w_{2}$, and for any $\gamma \in(0,1), \gamma w_{1}+(1-\gamma) w_{2}$ belongs to the relative interior of $D_{1}\left(q^{\prime}\right)$, i.e. there exists a open subset $\mathcal{O}$ of $\mathbb{R}^{d}$ such that

$$
\gamma w_{1}+(1-\gamma) w_{2} \in \mathcal{O} \cap \operatorname{Span}\left(D_{1}\left(q^{\prime}\right)\right) \subset D_{1}\left(q^{\prime}\right)
$$

which is always true for the classical isotropic Coulomb's friction characterized by a friction coefficient $\mu>0$.

\section{References}

1. Beghin, H.: Sur certains problèmes de frottement. Nouv. Ann. de Math., 2, 305-312 (1923-24)

2. Brogliato, B.: Nonsmooth mechanics, 2nd edition. Communications and Control Engineering, Springer Verlag, London (1999)

3. Coulomb, C.A.: Théorie des machines simples. Bachelier, Paris (1821)

4. Delassus, E.: Considérations sur le frottement de glissement. Nouv. Ann. de Math. (4ème série), 20, 485-496 (1920)

5. Dzonou, R., Monteiro-Marques, M.: Sweeping process for inelastic impact problem with a general inertia operator. Eur. J. Mech. A Solids, 26, 474-490 (2007)

6. Erdmann, M: On a representation of friction in configuration space. Int. J. Robotics Research, 13, 240-271 (1994)

7. Genot, F., Brogliato, B.: New results on Painlevé paradoxes. Eur. J. Mech. A Solids, 18-4, 653-677 (1999)

8. Lecornu, L.: Sur la loi de Coulomb. C.R. Acad. Sci. Paris, 140, 847-848 (1905)

9. Monteiro Marques, M.: Differential inclusions in non-smooth mechanical problems: shocks and dry friction. Birkhauser, Boston, Berlin (1993)

10. Moreau, J.J.: Standard inelastic shocks and the dynamics of unilateral constraints. In: Del Piero, G., Maceri, F. (eds.) Unilateral problems in structural analysis. C.I.S.M. Courses and Lectures, vol. 288, pp. 173-221. Springer, New York (1985)

11. Moreau, J.J.: Dynamique de systèmes à liaisons unilatérales avec frottement sec éventuel, essais numériques. Preprint 85-1 second edition, LMGC Montpellier (1986)

12. Moreau, J.J.: Unilateral contact and dry friction in finite freedom dynamics. In: Moreau, J.J., Panagiotopoulos, P.D. (eds.) Nonsmooth Mechanics and Applications. C.I.S.M. Courses and Lectures, vol. 302, pp. 1-82. Springer, New York (1988)

13. Painlevé, P.: Sur les lois du frottement de glissement. C.R. Acad. Sci. Paris, 121, $112-115(1895)$ 
14. Painlevé, P.: Sur les lois du frottement de glissement. C.R. Acad. Sci. Paris, 141, 401-405 and 546-552 (1905)

15. Paoli, L.: Vibro-impact problems with dry friction - Part I: Existence result to appear in SIAM Journal on Mathematical Analysis, 47-5, 3285-3313 (2015)

16. Paoli, L.: Multibody dynamics with unilateral constraints and dry friction: how the Contact Dynamics appraoch may handle Coulomb's law indeterminacies? to appear in Journal of Convex Analysis, 23, (2016)

17. Schatzman, M.: A class of nonlinear differential equations of second order in time. Nonlinear Anal. Theory, Methods and Applications, 2, 355-373 (1978) 